\title{
Genetics advances and learning disability ${ }^{\dagger}$
}

WALTER J. MUIR

\begin{abstract}
Background Medicine is rapidly becoming molecular medicine, and little escapes the grasp of modern genetics. Most disorders associated with learning disability have at least a genetic component influencing their expression; in many disorders, disturbances of genetic mechanisms play a pivotal role.
\end{abstract}

\begin{abstract}
Aims Dynamic mutations, imprinting mechanisms and gene-dosage effects are explained with reference to genetic disorders that lead to learning disability.
\end{abstract}

Method A review of recent important studies in the genetics of learning disability.

Results A host of new genetic connections to conditions associated with learning disability have been made.

Conclusions A basic understanding of these genetic connections is important for all learning disability psychiatrists if they are to follow the rapid changes - already beginning to influence our practice - that hold immense promise for the future.

Declaration of interest None.
Learning disability is a descriptive concept, not a disorder. A long held (and totally valid) view has been that the conditions leading to severe and profound learning disability have identifiable pathologies, including genetic disorders. Mild to moderate learning disability by contrast has been seen as largely sociocultural and multi-factorial/ polygenic in origin. However, an increasing number of single-gene conditions and subtle chromosomal rearrangements that lead to a mild to moderate outcome are known, and it is important for people with all degrees of learning disability that we discover as much as possible about the causal relationships involved.

\section{LEARNING DISABILITYAND GENETICS}

Our genome can be altered in many ways. The term 'mutation' usually implies change in the nucleotide sequence of single genes, altering their coding information. Mutation is one of three vital processes - the others being recombination and random chromosome assortment at fertilisation - that lead to the molecular individuality of a person. Mutations can be advantageous or harmful, depending on how they adapt the person for a particular environment. Single nucleotide changes (point mutations) can be very deleterious, especially if they occur in key developmental genes. Point mutations in the human eye-brain development gene 'sonic hedgehog' can lead to the disastrous outcome of holoprosencephaly. Mutations in its Drosophila larval counterpart lead to spiky outgrowths (hence the name). Such nomenclature now gives us desert, Indian, echnida, even tiggywinkle hedgehog genes (Johnson \& Scott, 1998). Many conditions previously thought to involve large numbers of genes (polygenic conditions) have now been shown to involve one gene or a few major genes (oligogenic conditions), and many seemingly unitary clinical conditions are genetically heterogeneous (e.g. tuberous sclerosis). On a larger scale, many genes can be affected by structural abnormalities of chromosomes. Duplications, with associated effects on gene dosage, can range from small partial trisomies through to the complete trisomies.

Karyotyping now involves molecular cytogenetics, with high resolution banding and fluorescent in situ hybridisation (FISH) revealing new sub-microscopic chromosomal rearrangements well below the classical banding levels (Flint et al, 1995). Deletions can be too small to detect by classical methods (examples, include many of the contiguous gene syndromes, where a microdeletion removes several genes), or large enough to cause complete chromosome arm loss (e.g. cri-du-chat) or whole chromosome loss (e.g. Turner's syndrome). Other rearrangements can disrupt gene structure directly or act by positional effects.

Familial disorders associated with learning disability often do not show simple Mendelian inheritance. Partial penetrance, parent-of-origin effects and anticipation occur - and in some cases (e.g. fragile-X) occur together. Advances in our understanding of the molecular mechanisms involved have been directly due to the study of disorders associated with learning disability, adding important new concepts such as dynamic mutations, imprinting and uniparental disomy to human genetics in general (the meaning of these terms will be explained below).

The fact that there are more men than women with learning disability has been known for over a hundred years, and is mainly due to $\mathrm{X}$-linked disorders. The $\mathrm{X}$ chromosome is also the best-studied human chromosome, with detailed genetic maps available. It is unsurprising then that a series of conditions associated with learning disability has been regionally mapped to the $\mathrm{X}$ chromosome. Another major advance has been the generation of new animal models useful in studying the neurological effects of gene mutations. The advent of transgenics, with its ability to knock out specific genes or insert specific human genes in (usually) the lab mouse, and to alter genes in targeted ways, has revolutionised our ability to correlate genotype and phenotype. Using such methods, a surprising amount of information has been gleaned about genetic conditions that produce learning disability.

It would be impossible to cover, even superficially, all recent genetic findings. 
Instead, the focus will be on areas of current interest that reveal how molecular genetics contributes to our understanding of the conditions associated with learning disability. The application of new methods to well-described conditions will centre on Down's syndrome, and dynamic mutations will be illustrated by fragile-X, while on the $\mathrm{X}$ chromosome several other key $\mathrm{X}$ linked conditions associated with learning disability will be discussed. Mention will be made of neuronal migration disorders located on autosomes, while the PraderWilli and Angelman syndromes will illustrate parent-of-origin effects, imprinting and uniparental disomies. Contiguous gene syndromes will focus on chromosome 22 deletions, which may partly explain the greatly increased rate of psychotic disorders in learning disability. Finally, there will be a brief discussion of where developments are leading in this field.

\section{DOWN'S SYNDROME: NEW INSIGHTS INTO TRISOMIES}

Down's syndrome is the paradigm of a (usually) non-inherited genetic disorder, where the effects are thought to be due to an excessive level of gene transcription (the dosage effect). To the psychiatrists its clinical presentation should need no description.

The density of genes on a chromosome plays a large part in determining whether a foetus with a particular trisomy will survive to term, and the banded morphology of stained chromosomes is now thought to have a definite functional basis. Genes cluster in poorly staining R-bands, especially in early-replicating R-bands termed T-bands. Certain chromosomes, such as 19 , are very T-band rich, others of comparable size such as 18 are comparatively gene poor. The three most survivable trisomies $(21,13$ and 18) are precisely those with the lowest chromosomal gene densities. An average chromosome, however, will contain about 4000 genes; even a very fine chromosome band of 3000000 base pairs ( $3 \mathrm{Mbp}$ ) may contain over 100, and almost certainly one of relevance to brain function. Gene clustering is relevant to the concept of a critical region for a condition. Rare individuals have chromosome 21 material in partial trisomy through a duplication or an unbalanced reciprocal translocation. Relating the gene content of this excess material to clinical phenotype may help the detection of specific genes producing specific clinical features, although some features may be due to the interactive effects of different genes in triplicate rather than simply to increased dosage of single genes. Some of these partial trisomies lie below the level of detection using classical banding methods. An example is shown in Figure 1.

Animal models for Down's syndrome, involving partial or full trisomy of the animal chromosome syntenic for human chromosome 21, have not always been convincing. Recently, however, transgenic mice have been created with an excess of human chromosome 21 material inserted into their genomes. By inserting different stretches of human chromosome 21 DNA, the phenotypic effects of regional trisomy can be explored (Smith et al, 1997). One such mouse displaying specific learning deficits was trisomic for a region containing the human form of the Drosophila minibrain gene. This gene immediately assumes a candidate role in human brain development, although there will undoubtedly be other genes in this region involved in neurological functioning. Such techniques should help the molecular dissection of other trisomies and disorders with a suspected dosage effect.

Another genetic aspect of Down's syndrome, one that continues to throw up puzzles, is the association with dementia. The role of Alzheimer precursor protein (APP) is unclear, but some mechanism must exist to explain why pathology does not usually appear until the third decade, even though APP levels are persistently high from foetal life. One suggestion is that an APP-scavenging mechanism fails with age, leading to plaques. The situation regarding proposed modifying genes is also confused. One finding is that the apolipoprotein $\varepsilon 4$ (APO- $\varepsilon 4)$ genotype may not have a direct influence on the causation or course of

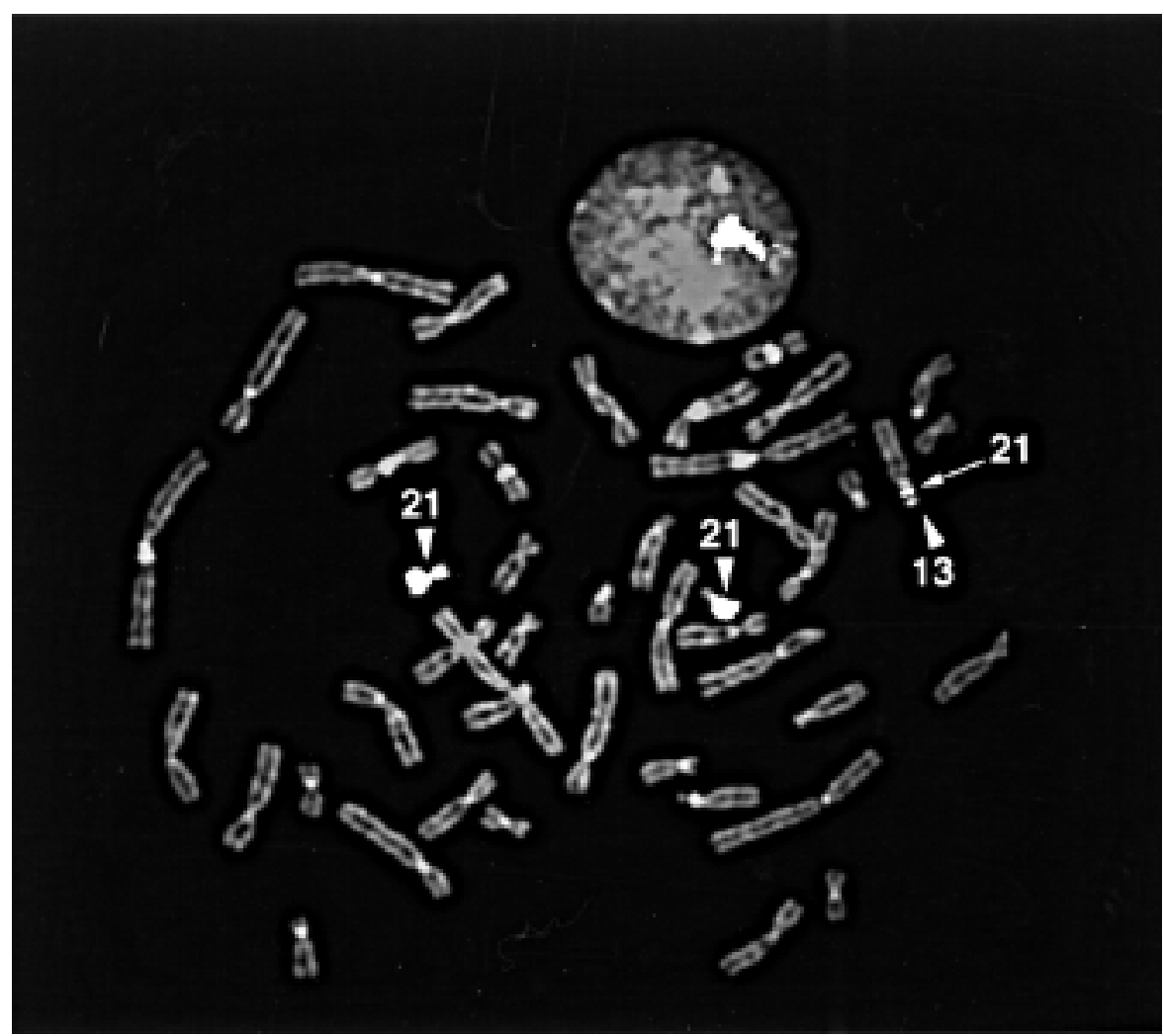

Fig. I Black and white reproduction of chromosome 2I fluorescent in situ hybridisation (FISH) painting on a metaphase from a patient with clinical Down's syndrome but with no abnormality on classical karyotyping. FISH was performed using a commercial flow-sorted whole chromosome 21 paint library conjugated with biotin, counterstained with 4,6-diamidino-2-phenylindole. Backgound and repeat sequence binding was eliminated using total human cot-I DNA and human ribosomal DNA. The chromosome banding pattern can been seen, as can the signal on chromosomes $2 \mathrm{l}$. There is also an interstitial band of chromosome $2 \mathrm{l}$ signal near the centromere of chromosome 13, which represents the small partial trisomy in this patient. An interphase nucleus (also showing chromosome $2 \mathrm{I}$ hybridisation) is present above of the metaphase. 
Alzheimer's disease, but may instead influence the age at which the disease is likely to occur or progress (Meyer et al, 1998). This finding may be relevant to age of onset in Down's syndrome, where recent findings indicate that individuals may be clinically spared - if they live long enough, dementia will develop.

\section{PRADER - WILLI AND ANGELMAN SYNDROMES: DELETIONS, UNIPARENTAL DISOMIES AND IMPRINTING MECHANISMS}

Prader-Willi syndrome, although not common (one in 10000-20 000 live births), has gained a number of notable firsts: it is the first human microdeletion syndrome found by high-resolution banding, the first human disorder where imprinting has been shown to play a critical role, and the first human condition where uniparental disomy contributes to its genesis (Cassidy, 1997). It also is the paradigm of a behavioural phenotype with developmental delays leading to variable degrees of learning disability, hyperphagia with severe obesity, hypogonadism and mild dysmorphic features such as small hands and feet and short stature. The study of such behavioural phenotypes, where specific reproducible constellations of behaviours are assumed to be conditional on an underlying common genotype, is becoming an important discipline in itself. In Angelman syndrome (which affects one in 30000 newborn babies), moderate to severe learning disability is associated with an ataxic gait, paroxysmal laughter, minimal speech, hand flapping and seizures.

The usual cause $(70 \%)$ of Prader-Willi syndrome is a microdeletion at chromosome 15q11-q13. The breakpoints are relatively stable and define a region about $4 \mathrm{Mbp}$ in length. Although the microdeletion is just visible on high-resolution banding, the diagnosis is now usually based on FISH. Detailed mapping using rare familial translocations and other rearrangements has shown that the critical regions for Prader-Willi syndrome and Angelman syndrome abut one another but do not overlap; the latter's critical region is more telomeric (closer to the end of the chromosome). In the normal person the PraderWilli syndrome region is known to contain at least seven gene sequences (including the neuronal necdin gene) solely expressed from the paternally derived chromosome 15. A deletion on the paternally derived chromosome 15 silences these genes, and Prader-Willi syndrome results. No singlegene mutations that produce Prader-Willi syndrome have been found; the syndrome is likely to be a true contiguous gene syndrome involving several genes. In Angelman syndrome the situation seems simpler. Again, deletions predominate (causing about $70 \%$ of cases) but this time on the maternally derived chromosome 15 . One gene, UBE3A coding for E6-APubiquitin protein ligase, is expressed from both chromosomes in most tissues, but preferentially from the maternally derived chromosome 15 in the human brain. Single point-mutations in this gene produce full clinical Angelman syndrome, and it may therefore be a true single gene disorder, although other genes may modify its expression (Jiang et al, 1998).

Another mechanism producing PraderWilli syndrome (30\%) and Angelman syndrome $(5 \%)$ is uniparental disomy. Here, both chromosome 15 s have apparently derived from one parent alone. A trisomy will contain two chromosomes from one parent and a third from the other. If one chromosome is lost the complement reverts to a disomy, and in one-third of cases this will contain two chromosomes from the same parent. Another (rarer) mechanism could be a monosomy that is followed by duplication of the chromosome. In the case of two chromosome $15 \mathrm{~s}$ arising from the mother, there would be a functional silencing of paternally expressed gene, resulting in Prader-Willi syndrome. Similarly, if both chromosomes originated from the father a functional silencing of maternally expressed genes would lead to Angelman syndrome. Uniparental disomy is usually detected by testing chromosome-15-specific polymorphic markers on DNA from the person with Prader-Willi syndrome or Angelman syndrome and both their parents. There is some evidence that the facial features of Prader-Willi syndrome caused by uniparental disomy are less prominent than those of Prader-Willi syndrome caused by deletion, so the degree of gene silencing may differ.

Studying why such genes are only expressed from a paternally or maternally derived chromosome has shed much light on the control of gene expression and has revealed a completely new variety of molecular disorder - imprinting centre mutations. Genomic imprinting (Constància et $a l, 1998)$ is the mechanism by which only one copy of a gene - that inherited from a particular parent - is activated or repressed. It is an epigenetic mechanism of controlling gene expression, since the underlying DNA sequence is not in itself disturbed and the changes are potentially reversible. One way to form an imprint is by the enzymatic addition of methyl groups to nucleotides in the DNA sequence. The classical instance is the large-scale methylation and packaging of the second $\mathrm{X}$ in women. However, specific differential methylation patterns of restricted regions of autosomal DNA are well known, and the clustering of genes that are methylated on one chromosome but not the other (as happens in the Prader-Willi syndrome/Angelman syndrome region) suggests a functional relationship between the different genes of the group. To establish a parent-specific imprint, in the first place the imprint from the previous generation must be cleared in the parents' germ-line. The imprint is removed from both chromosomes of the pair early in germ-cell development; later (perhaps around or even after birth), parent-genderspecific differential methylation occurs. Methylation of DNA sequences may alter (usually suppress, sometimes enhance) the binding of transcription or other factors controlling gene expression. Most human genes (about 60\%) reside under CpG islands - stretches of DNA enriched in C and $\mathrm{G}$ dinucleotides. Methylation of these islands usually silences the relevant genes, and $\mathrm{CpG}$ stretches are common in differentially imprinted regions. In the Prader-Willi syndrome/Angelman syndrome region, one paternal-chromosome expressed gene, SNRPN, is very tightly regulated by methylation, so that its promoter region and associated $\mathrm{CpG}$ island are completely differentially methylated in all somatic tissues. Its main product is a nuclear-ribonucleoprotein-associated polypeptide. Tiny microdeletions in the immediate vicinity of the SNRPN gene have been shown to alter the methylation pattern of the Prader-Willi syndrome/Angelman syndrome region and cause the relevant syndrome to occur. Such imprinting 'mutations' are rare (occurring in under $5 \%$ of the sufferers of either syndrome), but point to the existence of an imprinting centre involved in imprint establishment. Imprinting mutations leading to Angelman syndrome lie centromeric to those causing Prader-Willi syndrome, and both lie just centromeric to SNRPN, suggesting that the imprinting centre may have two functional parts, one relating to 
a paternal-to-maternal imprint switch and the other to a switch in the opposite direction. In the case of mutations producing Angelman syndrome, the critical part of the imprinting centre actually overlaps the SNRPN gene, and certain exons of SNRPN in this region encode a transcript with a function different from that of its main transcript. Whether this is involved in imprint formation is uncertain, but it shows expression specific to the paternally derived chromosome in the normal brain. The PraderWilli syndrome imprinting centre also overlaps the SNRPN gene promoter, giving this gene a possible accessory role in both types of imprint switch. Some imprinting centre microdeletions are inherited. In PraderWilli syndrome the patient has an imprinting centre mutation on the chromosome 15 derived from the father. The father is phenotypically normal but has the imprinting centre mutation of the chromosome 15 derived from his phenotypically normal mother, who also has a microdeletion (presumably arising de novo here). From the patient's point of view, both chromosome 15 regions are maternal in their imprint and there has been a failure of imprint switching in the father. In inherited Angelman syndrome imprinting mutations, the opposite holds true.

Three type A $\gamma$-aminobutyric acid receptor $\left(\mathrm{GABA}_{\mathrm{A}}\right)$ genes occur in the telomeric part of the Prader-Willi syndrome/ Angelman syndrome region. Their imprinting status is uncertain, but deletion of one (GABRB3) has been said to increase the severity of epilepsy associated with Angelman syndrome (Jiang et al, 1998), and duplications of the area have been implicated (Schroer et al, 1998) in some cases of autism. The incidence of psychosis in adults with Prader-Willi syndrome $(5 \%)$ is raised relative to the general population rate, and it would be important to see whether these people also had changes in their GABA receptors.

\section{THE X CHROMOSOME AND ITS DISORDERS}

Gender-linked disorders are very important in learning disability, and the $\mathrm{X}$ chromosome has been the most studied part of all the human genome.

Only one $\mathrm{X}$ chromosome must be fully active at one time in any diploid cell. Inactivation of the second $\mathrm{X}$ chromosome in women is a random process, controlled by an $\mathrm{X}$ chromosome inactivation centre (Xic). Within the Xic is a gene termed Xist, encoding an untranslated structural RNA that coats the $\mathrm{X}$ chromosome, which is then inactivated. The $\mathrm{X}$ chromosome seems to be rich in inactivation promoting signals, but inactivation will spread in a patchy fashion across any autosome translocated onto it. The active $\mathrm{X}$ chromosome does not express the Xist RNA, whereas the inactive $\mathrm{X}$ chromosome continues to do so. Xist gene deletions completely prevent inactivation, but DNA deletions in the immediate vicinity of the Xist gene cause non-random inactivation on the deletion chromosome only (Clerc \& Avner, 1998). Such sequences may be important in the counting process, in which inactivation occurs only if there are two or more Xics (this maintains $\mathrm{X}$ chromosome activity in Turner's syndrome). It would be interesting to study Xic effects in other sex chromosome anomalies. XXX, XXY and XYY karyotypes are relatively common (about one per 1000 live births for each). Subjects with Klinefelter's syndrome and XXX have lowered mean IQs; many therefore fall into the learning disability range. The $\mathrm{X}$ chromosome is unusual in that most of its genes show hemizygous expression (from only one chromosome). However, at least 20 genes, scattered throughout the short and long arms, escape inactivation; the spreading inactivation must therefore recognise regional or individual gene environments. A number of such genes cluster in one pseudo-autosomal region on the tip of the short arm, and another near the tip of the long arm. These regions show recombination within $\mathrm{XX}$ or $\mathrm{XY}$ pairs, and genes here are functionally expressed from both $\mathrm{X}$ and $\mathrm{Y}$ chromosomes. Hence, any clinical phenotype in sex chromosome polysomies is presumably due to dosage effects of duplications of the pseudo-autosomal and other genes that escape silencing. Indeed (excluding the fairly common mosaic karyotypes with two or more independent clonal cell lines), the higher the sex chromosome count, the greater the degree of learning disability (in, for example, tetrasomy and pentasomy $\mathrm{X}$ ). These conditions are an obvious target for future molecular study.

\section{Dynamic mutations}

Many inherited disorders do not obey Mendel's rules. Variable penetrance whereby gene disruption confers an increased susceptibility, not a $100 \%$ risk for a disorder - is common. Sometimes the disorder is more severe in the younger generations of a family - a phenomenon termed anticipation (historically, this term meant an earlier age at oneset; its present connotation is more general). In other cases there is a dependence on whether the disorder is inherited through the male or female germ line (the parent-of-origin effect). Most of our understanding of such patterns has come from the study of specific forms of learning disability, and one essential step was the discovery of a dynamic unstable repeat element and associated gene (FMR-1) underlying fragile-X syndrome.

Fragile-X is a relatively common cause of learning disability (affecting about one in 4000 men and one in 8000 women); it shows anticipation, the parent-of-origin effect, and a very low apparent penetrance compared with other X-linked disorders. The phenotype is variable, the most consistent features being learning disability and, in affected men, macro-orchidism. Before the discovery of the gene, diagnosis rested on phenotype, family history, and a karyotype artefact - a constriction at Xq27.3 (a fragile site now termed FRAXA) in a percentage of metaphases when the culture medium is deprived of folate. The degree of learning disability is usually moderate in affected men and more variable in women; a mild degree may also exist in some female carriers.

A stretch of DNA composed of a repeated triplet of nucleotides (CCG) exists in the $5^{\prime}$-untranslated end of the FMR-1 gene. Men affected with fragile-X have a large expansion of $230-1000$ or more triplets. The cytosine residues of CG base pairs are highly methylated here and in the surrounding DNA areas, including the CpG island/promoter region essential to transcription factor binding-hence the gene is silenced. Rare individuals with specific deletions or silencing point-mutations of FMR-1 have full clinical fragile-X, indicating that disruption of FMR-1 alone is enough to produce the condition. With the dynamic mutation it is the methylation that is important rather than any direct effect on gene transcription, since unusual enzyme defects that prevent gene methylation do not result in fragile-X even if a full expansion is present. Furthermore, experimental demethylation of the gene restores full transcriptional activity (Chiurazzi et al, 1998). 
In the general population the repeat consists of 6-50 triplets (mean 30). These can be passed on from one generation to the next without phenotypic consequences or change in their size. Triplets are mainly CGG nucleotides, but about one in 10 is AGG. A relative lack of AGG sequences (increasing the run of 'perfect' CGG triplets) or an increase in the number of CGG triplets towards the $3^{\prime}$ end of the sequence introduces length instability, increasing the risk of a small expansion in size when passed on in the normal population. This may be one source of a slow increase in the size of small repeats in the general population. In the families of fragile-X patients the situation is different. 'Premutations' of 60-200 triplets occur in the mothers and in men who must be transmitting the disorder ('normal transmitting males'). When transmitted by a mother to her child, these premutations are unstable and almost invariably undergo a large expansion (further CGG triplets are added). Smaller premutations may expand by maternal transmission to large premutations, and large premutations may expand to the very large mutation seen in individuals with full fragile-X. Like the normally sized repeat, the breakage of a pure run of CGGs by AGG stabilises the premutation (and is another source of variance in the clinical counselling of this condition).

The conversion of an unstable premutation into a large expansion by passage through a female meiosis causes the parentof-origin effect. The mechanism of the expansion is uncertain, but interestingly it is not present in the sperm of men with full fragile-X. Either the germ line is protected from full expansion, or sperm with the expansion are selectively eliminated, or there is a true regression in size of the expansion. Work from Oostra's group (Matter et al, 1997) favours the last explanation, the further study of which may help us to understand the mechanisms involved in changing an expansion's size. Whichever explanation is correct, there must be some imprinting mechanism specific to female transmission that differentiates a premutation from a normally sized repeat. Furthermore, in fragile- $\mathrm{X}$ there is high incidence of somatic cell mosaicism for both full mutations, and premutations, and the premutation-carrying cells are transcriptionally active, making exact genotype-phenotype correlations very difficult. The FMR-1 mutation produces an RNA binding protein (FMRP, Fragile-X Mental Retardation
Protein) with the widespread tissue distribution that may play a role in cellular mRNA transport. The highest FMRP levels occur in the brain (in the cortex and especially the cerebellum) and testes. The brain in fragile-X shows increased size of the hippocampal and caudate nucleus and decreased size of the posterior cerebellar vermis. The latter may also be reduced in some people with autism, and links between the conditions have been postulated.

The repeat's population genetics has also been studied. In some populations (e.g. in Scandinavia) the new mutation frequency is low, with evidence for an ancestral founder mutation, a feature unusual in an X-linked condition. In the UK the haplotype diversity is wider, with less evidence for a founder effect. Such population differences in origin and carriage may imply that different processes are responsible for the upward creep in size. A large Canadian study (Rousseau et al, 1995) found repeats of 66 or more triplets in one in 500 women and a similar frequency for 55-63 triplet repeats, and indicated that around one in 2900 children should carry the mutation. Detection and sizing of the repeat are now carried out on DNA extracted from a venous blood sample, but many subjects (particularly older men with learning disability) remain unclassified, correcting this will be important, especially if the subjects have phenotypic features or a family history.

Around 12 other dynamic mutations are known (Richards \& Sutherland, 1997). The $X$ chromosome (Xq28) fragile site FRAXE harbours a CGG repeat whose expansion and associated gene silencing (FMR2 - the protein is expressed in the human brain, including the hippocampus) produce mild learning disability or specific educational problems. However, the repeat in this case can expand or contract on transmission. Dynamic mutations need not be restricted to triplets. A common polymorphic repeat of $33 \mathrm{bp}$ is found in FRA16A, so far without an associated clinical phenotype; and many more probably remain to be discovered.

\section{The LI-associated conditions: one gene, many clinical conditions}

The genetics of learning disability is the genetics of neurodevelopment. Developing neurones are controlled and guided in their physical migrations, in their axon and dendrite tree spreading, and in the eventual establishment or synaptic connections, by complex cellular signalling mechanisms involving diffusible factors or direct cellcell contacts. An important set of proteins mediating the latter is the L1 group of neural cell adhesion molecules. Structurally resembling immunoglobulins, these molecules act as both adhesion molecules and signalling receptors. Human L1 gene again lies on the $\mathrm{X}$ chromosome (Xq28); mutations cause a wide spectrum of important neurological conditions, often with severe learning disability. The first association made was with $\mathrm{X}$-linked hydrocephalus, but mutations in the same gene can cause complicated spastic paraparesis (type 1), corpus callosum agenesis, and MASA (mental retardation-aphasia-shuffling gaitadducted thumbs) syndromes. These have been grouped under the acronym CRASH syndrome (corpus callosum, mental retardation, adducted thumbs, spastic paraplegia, hydrocephalus). Over 85 different mutations have been described in this gene alone, and the particular type of mutation relates to clinical outcome (Fransen et al, 1998). Coding region mutations in the extracellular domain of L1 cause truncation or absence of the protein, leading to an extreme phenotype (severe hydrocephalus, profound learning disability, often early mortality). Cytoplasmic domain mutations, by contrast, may only cause partial loss of functioning and a much milder, variable disorder without ventricular dilatation. The clear relationship between genotype (mutation position within the gene structure) and clinical phenotype is fascinating but does not allow us, in itself, to understand the developmental pathways involved in the different outcomes. However, mouse models with complete knock-out of the L1 gene correspond to severe phenotype mutations (Kamiguchi et al, 1998), and targeted mutagenesis should allow the creation of specifically altered L1 genes that model the other phenotypes.

\section{The non-syndromic $X$-linked conditions: one clinical condition, many genes}

Many families with multiple individuals affected by learning disability seem to fit into an X-linked inheritance pattern. Nevertheless, imaging and other investigations show that gross anatomical brain development in these individuals is indistinguishable from that of the normal population, and there are few symptoms other 
than learning disability. Clinically, these conditions are homogeneous, and they have earned the title of 'non-syndromic X-linked mental retardation'. Sufferers form an important subset of people with learning disability $(0.3 \%$ men), and behind the clinical similarity lies a great deal of genetic heterogeneity. FRAXE (see above) was the first such condition to be understood, but several other non-syndromic X-linked mental retardation genes have now been found. One gene (GD11, Xq28) may modulate exocytosis during synaptic transmission. A second gene at $\mathrm{Xq} 12$ produces a protein (unfortunately) labelled as oligophrenin (Billuart et al, 1998). The clue to finding this gene was a female with mild learning disability with a $t(X ; 12)$ balanced reciprocal translocation where the breakpoint had disrupted the gene's intron/exon structure. Different loss-of-function mutations in this gene were found in other unrelated individuals. The protein, a rho-GTPase, may influence signalling during cell migration and axon-dendrite outgrowth. Most recently, a series of brain-expressed genes has been identified within Xq21-q24 one of which, PAK3, is mutated in all affected males in a family with nonsyndromic X-linked mental retardation, with carrier females being heterozygotes (Allen et al, 1998). The PAK3 protein, also part of the rho-GPTase signalling chain, is highly expressed in the developing cerebral cortex and hippocampus. It is certain that many more genes will be discovered, each with its own different set of mutations.

\section{AUTOSOMES AND DISORDERS OF NEURODEVELOPMENT}

The lissencephalies: disorders of neuronal migration

Brain sulci and gyri are underdeveloped in many syndromes associated with learning disability, such as Down's syndrome. However, the most striking degree of underdevelopment occurs in the lissencephalies (the 'smooth brains'), where arrested neuronal migration in which the normal sixlayered folded cortex does not develop leads to a profound degree of learning disability. One human lissencephaly gene (the unpronounceable PAFAH1B1 on chromosome $17 \mathrm{p}$ ) is disrupted by a hemizygous microdeletion in Miller-Dieker syndrome. Since the deletion is on one chromosome only and the gene is usually expressed from both chromosomes, the effect is due to a reduction in protein level rather than absence. A mouse hemizygous knockout model has been created and has thrown light on many aspects of neural migration (Hirotsune et al, 1998), but does not show the severe Miller-Dieker syndrome cortical phenotype, serving to remind us that mouse is not always akin to man. Another (X chromosome) lissencephaly gene produces the protein doublecortin, which may act on migration through intracellular calciumdependent signalling (Sossey-Alaoui et al, 1998). Clinically its effect is very similar to Miller-Dieker syndrome.

\section{Microcephaly}

Microcephaly is an extremely heterogeneous clinical condition, with a variety of intrauterine toxins, infections and birth asphyxia being implicated in individual cases. However, true recessive microcephaly is an autosomal-recessive disorder presenting simply with microcephaly and learning disability without other severe neurological features. It has been analysed in two consanguineous kindreds by a whole-genome linkage scan using the method of homozygosity mapping (Jackson et al, 1998). In essence, data are obtained from a large number of closely spaced polymorphic markers covering all chromosomes, and identical stretches of marker allelles (haplotypes) that are present on both chromosomes of a pair are identified in affected individuals. Recessive disorders require disruption of genes on both chromosomes of a pair, the smallest interval of such two-chromosome haplotype-identity shared between different affected family members defines the region proposed to harbour the gene of interest, in this case on chromosome 8q.

\section{CONTIGUOUS GENE SYNDROMES: DISORDERS OF MANY GENES AT ONCE}

Fluorescent in situ hybridisation (FISH) methods, where a DNA probe(s) is directly or indirectly coupled to a fluorescent reporter element, have revolutionised karyotyping. The previous resolution limit with high-resolution banding was about $3 \mathrm{MbP}$; FISH can detect much smaller changes (one method - fibre FISH - teases out uncoiled interphase chromosomes and can detect differences in composition at the kilobase level). A whole series of new and subtle chromosomal changes has been unveiled, along with an increasing number of interstitial microdeletion and duplication syndromes producing contiguous gene syndromes. Many of the latter have a related learning disability, while all involve the disruption of a series of grouped genes by the abnormality. Prader-Willi syndrome is a contiguous gene syndrome that shows imprinting effects, as does BeckwithWeidemann syndrome (duplication of 11p15). Most contiguous gene syndromes are deletions with reductions in gene dosage (haploinsufficiency); examples are Miller-Dieker (17p13.3), Langer-Giedon (8q24.1) and Rubenstein-Taybi (16p13.3) syndromes.

\section{The diGeorge and velo-cardio- facial syndromes}

These clinically overlapping disorders are associated with a microdeletion at chromosome $22 \mathrm{q} 11$ that can be detected using FISH. They are frequent conditions (one in 4000 live births) occurring, for example, in $5 \%$ of children with congenital heart defects. The pattern of anomalies suggests abnormal movement of neural crest cells that migrate into the embryonic pharyngeal arches. DiGeorge syndrome is associated with micrognathia, cleft palate, low-set ears, hypertelorism, short palpebral fissures and major cardiac abnormalities. Velocardio-facial syndrome is extremely variable (symptoms include midface underdevelopment, bulbous nose, cleft palate, neonatal parathyroid and thymus problems, and conotruncal cardiac abnormalities), but has a fairly consistent mild learning disability. The variability occurs between individual sufferers and even within the same family. Deletions with different breakpoints have helped to define the critical region of $480 \mathrm{kbp}$, from which seven genes have so far been isolated; the latest, which is expressed in many human tissues including foetal and adult brains and head and neck (Funke et al, 1998), is a key candidate for neuropsychiatric effects.

There has recently been interest in the association between these syndromes (and $22 \mathrm{q} 11$ deletions) and the risk of developing schizophrenia and learning disability (Murphy et al, 1998). Deletions have been claimed in one in 40 of those with both diagnoses and in one in 200 of those with schizophrenia alone. Independent studies have also suggested linkage between polymorphic DNA markers in this region and 
familial schizophrenia. The fact that the point-prevalence of schizophrenia in people with mild learning disability is three times that of the general population is well known, and comorbid subjects have recently been shown to have a striking incidence of family history, with a very variable phenotype in relatives (Doody et $a l, 1998)$. This familiality is unexplained, but chromosome abnormalities such as del(22) that co-associate with the condition may provide important clues in the search for genes. In fact, chromosome abnormalities have been pointers to the loci for genes for many genetic diseases, and the case is strengthened where there is additional independent evidence of linkage of the condition to the abnormal chromosome. One such case occurs where two separate families (Mors et al, 1997) segregating a pericentric inversion (18) with phenotypes of psychotic illness and learning disability have breakpoints at the two loci with strong independent evidence of linkage to both bipolar disorder and schizophrenia.

\section{THE FUTURE}

The polymerase chain reaction (PCR), a now well-established method, has changed the face of molecular genetics, allowing the specific amplification of DNA from tiny amounts of material, and has facilitated the international human genome sequencing effort. This project is generating massive quantities of new gene sequences that are candidates for conditions underlying learning disability. Methods permitting the economic screening of thousands of these genes are being devised. DNA chip-based assay is an emerging technology in which gridded microarrays of thousands of DNAs, cDNAs, PCR products and other nucleotide sequences can be attached to small pieces of glass or silicon and analysed for hybridisation matches with patient samples in a massively parallel fashion (Hacia et al, 1998). Some methods allow the arrays to be repeatedly re-probed, and multicolour fluorescent reporter systems can be used to distinguish homozygous and hemizygous gene changes. With a new, highly abundant category of polymorphic markers (the single nucleotide polymorphisms), chips could be used to screen for multiple sequence variations associated with complex diseases, and help to identify genes at work in multigenic disorders. Commercial

\section{CLINICAL IMPLICATIONS}

Genetic advances have revealed the molecular underpinnings of many conditions associated with learning disability. New diagnostic groupings based on genotypes will place developmental and clinical studies on a firmer biological basis.

- High-throughput molecular diagnostics will allow the screening of large numbers of individuals for learning disability conditions in future.

- The understanding of molecular mechanisms is likely to help the development of better targeted interventions and possible therapies.

\section{LIMITATIONS}

It has not been possible to cover all genetic conditions and mechanisms associated with learning disability in this review.

- Even with the advent of transgenics, animal genetic models do not always mirror the human condition.

- The rapid advances in understanding genetic mechanisms have not been matched by comparable developments in our thinking about ethical and moral issues.

WALTER J. MUIR, MRCPsych, Department of Psychiatry, University of Edinburgh, Kennedy Tower, Royal Edinburgh Hospital, Morningside Park, Edinburgh EHIO 5HF

(First received I2 July 1999, final revision 15 September 1999, accepted 16 September 1999)

interest in chip technology techniques is high and looks set to revolutionise molecular diagnostics.

We also need a revolution in our ethical thinking about the implications of these changes. To ignore advances would be therapeutic nihilism; however, moral and legal safeguards for patients and their families are urgently needed, as the pace of change is extraordinary. There is indeed a danger of creating a 'new eugenics', and the lessons of Hadamar should never be forgotten. All learning disability psychiatrists should read the report of the Nuffield Council on Bioethics (1998), where all issues, including insurance, employment and education, are discussed.

\section{ACKNOWLEDGEMENTS}

I would like to apologise to the multitude of authors whose work could not be referenced for lack of space. The FISH karyotype picture was prepared by Mrs Pat Malloy in a study supported by the Medical Research Council (MRC). Human ribosomal DNA for Fig. I was donated by Dr Wendy Bickmore, of the MRC Human Genetics Unit.

\section{REFERENCES}

Allen, K. M., Gleeson, J. G., Bagrodia, S., et al (1998) PAK 3 mutation in nonsyndromic $X$-linked mental retardation. Nature Genetics, 20, 25-29.

Billuart, P., Bienvenue, T., Ronce, N., et al (1998) Oligophrenin-I encodes a rhoGAP protein involved in X-linked mental retardation. Nature, 392, 923-926.

Cassidy, S. B. (1997) Prader-Willi syndrome. Journal of Medical Genetics, 34, 913-923.

Chiurazzi, P., Pomponi, M. G., Willemsen, R., et al (1998) In vitro reactivation of the FMRI gene involved in fragile X syndrome. Human Molecular Genetics, 7 109-113.

Clerc, P. \& Avner, P. (1998) Role of the region 3' to Xist exon 6 in the counting process of $X$-chromosome inactivation. Nature Genetics, 19, 249-253.

Constància, M., Pickard, B., Kelsey, G., et al (1998) Imprinting mechanisms. Genome Research, 8, 88I-900.

Doody, G. A., Johnstone, E. C., Sanderson, T. L., et al (1998) 'Pfropfschizophrenie' revisited. Schizophrenia in people with mild learning disability. British journal of Psychiatry, 173, 145-153.

Flint, J., Wilkie, A. O. M., Buckle, V. J., et al (1995) The detection of subtelomeric chromosomal rearrangements in idiopathic mental retardation. Nature Genetics, 9, 132-140.

Fransen, E., van Camp, G., D'Hooge, R., et al (1998) Genotype-phenotype correlation in $\mathrm{LI}$ associated diseases. Journal of Medical Genetics, 35, 399-404. 
Funke, B., Puech, A., Saint-Jore, B., et al (1998) Isolation and characterization of a human gene containing a nuclear localization signal from the critical region for velo-cardio-facial syndrome on 22qll. Genomics, 53, 146-154.

Hacia, J. G., Brody, L. C. \& Collins, F. C. (1998) Applications of DNA chips for genomic analysis. Molecular Psychiatry, 3, 483-492.

Hirotsune, S., Fleck, M. W., Gambello, M. J., et al (1998) Graded reduction of Pafah Ibl (Lisl) activity results in neuronal migration defects and early embryonic lethality. Nature Genetics, 19, 333-339.

Jackson, A. P., McHale, D. P., Campbell, D. A., et al (1998) Primary autosomal recessive microcephaly (MCHPHI) maps to chromosome 8p22-pter. American Journal of Human Genetics, 63, 54I-546.

Jiang, Y.-H., Tsai, T.-F., Bressler, J., et al (1998) Imprinting in Angelman and Prader-Willi syndromes. Current Opinion in Genetics and Development, 8 , 334-342.

Johnson, R. L. \& Scott, M. P. (1998) New players and puzzles in the hedgehog signalling pathway. Current Opinion in Genetics and Development, 8, 450-456.
Kamiguchi, H., Hlavin, M. L. \& Lemmon, V. (1998) Role of $\mathrm{LI}$ in neural development: what the knockouts tell us. Molecular and Cellular Neuroscience, 12, 48-55.

Matter, H. E., Iber, J. C., Willemsen, R., et al (1997) Characterization of the full fragile-X syndrome mutation in fetal gametes. Nature Genetics, I5, 165-169.

Meyer, M. R., Tschanz, J.T., Norton, M. C., et al (1998) APOE genotype predicts when - not whether - one is predisposed to develop Alzheimer's disease. Nature Genetics, 19, 321-322.

Mors, O., Ewald, H., Blackwood, D., et al (1997) Cytogenetic abnormalities on chromosome 18 associated with bipolar affective disorder or schizophrenia. British Journal of Psychiatry, I70, 278-280.

Murphy, K. C., Jones, R. G., Griffiths, E., et al (1998) Chromosome 22qll deletions. An under-recognised cause of idiopathic learning disability. British Journal of Psychiatry, 172, 180-183.

Nuffield Council on Bioethics (1998) Mental Disorders and Genetics: The Ethical Context. London: Nuffield Council on Bioethics.
Richards, R. I. \& Sutherland, G. R. (1997) Dynamic mutation: possible mechanism and significance in human disease. Trends in Biochemical Science, 22, 423-436.

Rousseau, F., Rouillard, P., Morel, M. L., et al (1995) Prevalence of carriers of permutation-size alleles of the FMRI gene and implications for the population genetics of the fragile-X syndrome. American Journal of Human Genetics, 57, 1006-1018.

Schroer, R. J., Phelan, M. C., Michaelis, R. C., et al (1998) Autism and maternally derived aberrations of chromosome 15q. American Journal of Medical Genetics, 76, 327-336

Smith, D. J., Steven, M. E., Sudanagunta, S. B., et al (1997) Functional screening of $2 \mathrm{MB}$ of human chromosome 21q22.2 in transgenic mice implicates minibrain in learning defects associated with Down syndrome. Nature Genetics, 16, 28-36.

Sossey-Alaoui, K., Hartung, A. J., Guerrini, R., et al (1998) Human doublecortin (DCX) and the homologous gene in mouse encode a putative $\mathrm{Ca}^{2+}$ dependent signalling protein which is mutated in human $X$-linked neuronal migration defects. Human Molecular Genetics, 7, 1327-1332. 